\title{
Are the current recommendations for the use of aspirin in primary prevention of cardiovascular disease applicable in low-income countries?
}

This article was published in the following Dove Press journal:

Vascular Health and Risk Management

25 August 2015

Number of times this article has been viewed

\author{
Jean Jacques $N$ Noubiap ${ }^{1,2}$ \\ Jobert Richie N Nansseu ${ }^{3,4}$ \\ 'Department of Medicine, Groote \\ Schuur Hospital, University of Cape \\ Town, Cape Town, South Africa; \\ ${ }^{2}$ Medical Diagnostic Center, Yaoundé, \\ Cameroon; ${ }^{3}$ Sickle Cell Disease Unit, \\ Mother and Child Centre, Chantal \\ BIYA Foundation, Yaoundé, Cameroon; \\ ${ }^{4}$ Department of Public Health, Faculty \\ of Medicine and Biomedical Sciences, \\ University of Yaoundé I, Yaoundé, \\ Cameroon
}

\begin{abstract}
Although evidence has accumulated that long-term aspirin therapy is beneficial in secondary prevention of cardiovascular disease (CVD), a lot of controversies persist regarding the benefit of aspirin use in primary prevention of CVD. In low-income countries (LIC) specifically, the decision to prescribe aspirin for primary CVD prevention is more problematic, as there is a dearth of evidence in this regard. Aspirin has been shown to have relative beneficial effects in preventing a first myocardial infarction, but not stroke. However, as stroke is the prevailing CVD in many LIC, especially in Africa, the benefit of aspirin in these settings is therefore questionable. Indeed, there is no published trial that has evaluated the benefits and risks of continuous aspirin therapy in populations of LIC. Furthermore, though cardiovascular risk assessment is crucial in decision-making for the use of aspirin in primary prevention of CVD, there are no risk assessment tools that have been validated in African populations. Studies are urgently warranted, to determine the usefulness of aspirin in primary prevention of CVD in low-income settings where the drug is highly available and affordable, as CVD is becoming the leading cause of deaths in LIC.
\end{abstract}

Keywords: aspirin, cardiovascular disease, primary prevention, low-income countries

\section{Introduction}

Cardiovascular disease (CVD) is becoming the leading cause of deaths in the developing world. Indeed, CVD is overtaking HIV infection, malaria, and tuberculosis as the leading cause of deaths in this part of the globe. ${ }^{1}$ The World Health Organization estimates that annual mortality due to CVD will approach 25 million by 2030 worldwide, of which approximately $80 \%$ will occur in low-income countries (LIC) and middle-income countries. ${ }^{2}$ While the rate of deaths from CVD has been increasing in LIC and middle-income countries since the mid-1970s, it has declined markedly in several high-income countries (HIC) during the same period, owing to a combination of successful population-wide preventive strategies, effective primary and secondary preventive health care, and improved treatment of acute cardiovascular events. ${ }^{3}$ Unfortunately, LIC, especially in the African region, which are still confronted with a high burden of infectious diseases, are meanwhile ill-prepared to cope with the rising epidemics of CVD. Hence, interventions that have been so successfully implemented in HIC to tackle the burden of CVD are still largely missing in most LIC health care services. ${ }^{4}$ Effective preventive treatments that are available at low cost, such as aspirin, are still underused by most individuals for whom such treatments can be recommended. ${ }^{5}$
Correspondence: Jean Jacques $\mathrm{N}$ Noubiap

Department of Medicine, Groote Schuur Hospital, University of Cape Town, J Floor, Old Main Building, Observatory, Cape Town, 7925, South Africa

Email noubiapji@yahoo.fr 


\section{Benefits and harms of aspirin in primary prevention of CVD}

Because of the prominent role of blood coagulation in the pathogenesis of acute vascular disease, the effectiveness of antithrombotic drugs, including aspirin, has been tested for the prevention of CVD. While there is overwhelming evidence that aspirin is beneficial in secondary CVD prevention, ${ }^{6}$ results from randomized clinical trials and meta-analyses on the benefits of aspirin in primary prevention of cardiovascular events are heterogeneous.

In 2009, the Antithrombotic Trialists' Collaboration (ATTC) conducted a meta-analysis of the first six primary prevention trials with aspirin including 95,000 participants, which showed that aspirin therapy led to a reduction of six myocardial infarctions (MIs) per 1,000 low-risk persons treated $(5 \%$ coronary heart disease [CHD] risk at 10 years according to the Framingham risk categories). ${ }^{7}$ Moreover, aspirin use was associated with a reduction of 19 MIs per 1,000 moderate-risk persons (15\% CHD risk) and $31 \mathrm{MIs}$ per 1,000 high-risk persons ( $25 \%$ CHD risk) over a 10 -year period. ${ }^{8}$ Aspirin therapy seemed to have no effect on stroke occurrence. ${ }^{7}$ With respect to mortality, there was a small protective effect of aspirin therapy, with zero to six fewer deaths per 1,000 persons treated over 10 years. $^{8}$ Additionally, a meta-analysis of nine clinical trials including 50,868 subjects found no overall benefit of aspirin for the primary prevention of stroke. ${ }^{9}$ Consistent with other studies, ${ }^{7,10}$ this meta-analysis reflected no benefit of aspirin use for the prevention of a first stroke in the general population.

Besides, bleeding is the most frequent adverse event associated with chronic use of aspirin. In fact, the ATTC meta-analysis revealed that aspirin increased the risk of major gastrointestinal and other extracranial bleeds by approximately $50 \%{ }^{7}$ Further, aspirin has been shown to increase the incidence of hemorrhagic stroke by $22 \% .{ }^{11}$ Mirroring ATTC results, De Berardis et al found that aspirin therapy was associated with an excess risk of gastrointestinal and intracranial bleeding. ${ }^{12}$ Likewise, in the recently published Japanese Primary Prevention Project survey, aspirin significantly increased the risk of extracranial hemorrhage requiring transfusion or hospitalization. ${ }^{13}$

Overall, despite the apparent benefits of aspirin in primary CVD prevention, these benefits may be outweighed by the risk of major bleeding. Indeed, a recent systematic review and overview of reviews of aspirin prophylaxis for primary prevention of CVD concluded that "there is a fine balance between benefits and risks from regular aspirin use in primary prevention of cardiovascular disease". ${ }^{14}$
Therefore, aspirin should be prescribed, based on weighing the absolute benefit in reducing the risk of the first cardiovascular event against the absolute risk of major bleeding in each patient. For example, aspirin can be considered in patients with a family history of MI at an early age or those with multiple cardiovascular risk factors, whereas it should be avoided in patients with risk factors for gastrointestinal bleeding such as dyspepsia and ulcer. ${ }^{15}$ Besides, the potential benefit of aspirin in cancer prevention could also be considered in patients with additional risks of neoplastic diseases, especially in cases of colorectal cancer. ${ }^{16}$

\section{Peculiarity of LIC}

Although more than $80 \%$ of the global burden of CVD occurs mostly in LIC, ${ }^{1}$ there is a paradoxical lack of local accurate data on the patterns of CVD and related risk factors in these countries. Furthermore and to the best of our knowledge, no studies have either investigated the benefits and harms of chronic aspirin use in LIC, especially those from the African region, or developed CVD risk assessment tools that are adapted to various geographical regions and ethnic groups. These gaps need to be urgently filled to achieve effective CVD prevention in these areas.

The difference in epidemiological patterns of CVD between HIC and LIC raises some concerns about the applicability of recommendations for aspirin use in primary prevention of CVD in LIC. Indeed, recommendations for chronic aspirin use in primary prevention of cardiovascular events have been released, mostly based on the benefits of aspirin for the prevention of the first MI, ${ }^{17} \mathrm{CHD}$ being the leading CVD in most HIC. ${ }^{17}$ Contrariwise, studies have shown the predominance of cerebrovascular disease over CHD in many LIC, especially in the African region. ${ }^{18}$ The absence of benefit of aspirin for the prevention of the first stroke in the general population in some HIC questions its overall benefit in primary prevention of CVD in populations where stroke is the prevailing CVD. ${ }^{7,9,10}$ Nonetheless, the Women's Health Study showed that taking daily low-dose aspirin significantly reduces the risk of the first ischemic stroke in women aged 45 years or older, ${ }^{19}$ suggesting that similar benefits could be observed in LIC with high burden of stroke. Robust evidence on the benefit of aspirin for primary prevention of CVD is therefore highly needed to inform adequate and efficient policies that are applicable in LIC.

Cardiovascular risk assessment is an important element in decision-making for the use of aspirin in primary prevention of CVD. Several cardiovascular risk assessment tools have been developed in HIC based on extensive high-quality 
epidemiological data. Such tools include the Framingham CHD risk score, the American College of Cardiology/ American Heart Association Task Force risk equations, the the European Society of Cardiology Systematic Coronary Risk Evaluation (ESC's SCORE), the coronary artery calcium score, among others. These assessment tools are very useful, although a recent study has shown that some of them may significantly overestimate the risk of atherosclerotic CVD. ${ }^{20}$ Besides, as most of these tools were developed in populations from HIC and predominantly Caucasians, their applicability in other populations or ethnic groups is therefore problematic. In this regard, the INTERHEART risk score was developed based on data from 52 countries around the world. ${ }^{21}$ Yusuf et al showed that the INTERHEART risk score, based on nine easily measureable and potentially modifiable risk factors, is associated with more than $90 \%$ of the likelihood to accurately predict an acute MI, these results being consistent across all geographical regions and ethnic groups of the world, men and women, and young and old ages. ${ }^{21}$ More recently, McGorrian et al modified the INTERHEART risk score into the INTERHEART modifiable risk score, of which four risk score models were derived. ${ }^{22}$ Of these, the non-laboratory-based score, which does not include any lab-based measures of lipid profile, can be used in primary care settings, especially in resource-poor countries where there is lack of laboratories and locally developed CVD risk assessment tools. ${ }^{4}$ However, it is worth emphasizing that this INTERHEART modifiable risk score has not been validated in African populations, ${ }^{22}$ hence the need for such validations.

Furthermore, there is body of evidence suggesting that the cardiovascular risk profile of populations in HIC may differ from that of populations in LIC. Indeed, a recent study investigating cardiovascular risk and events in 17 LIC, middle-income countries, and HIC revealed that although the INTERHEART risk score classifies residents of HIC as being at greater cardiovascular risk than residents of LIC, the rates of major cardiovascular events and deaths are substantially higher in LIC. ${ }^{23}$ This discrepancy was unexplained by the prevalence of traditional cardiovascular risk factors such as hypertension and diabetes, ${ }^{23}$ suggesting that traditional cardiovascular risk scores might underestimate cardiovascular risk among populations of LIC where nontraditional risk factors such as socioeconomic status, infectious diseases, and household and ambient air pollution may significantly impact the cardiovascular risk in these settings. ${ }^{24}$ This highlights the limits of the traditional cardiovascular risk assessment tools in LIC and stresses the need to develop assessment tools that are adapted to these contexts.
Finally, there are no current cost-effective alternatives to aspirin therapy in LIC. Data on availability and affordability of essential medicines in LIC showed that while aspirin is highly available and affordable in these areas, drugs such as clopidogrel and statins that have been presented as safe alternatives to aspirin have limited availability and are largely unaffordable. ${ }^{4,25}$ While waiting for cost-effective-and-benefit studies in LIC that will compare aspirin to other alternatives, continuous aspirin therapy for the primary prevention of CVD needs to be still considered and balanced against the potential for bleeding in each patient. Studies investigating the adherence and compliance to this long-term medication are also warranted.

\section{Conclusion}

The use of aspirin in primary prevention of CVD remains a topic of debate, especially in LIC where lack of data renders the adoption of such a strategy highly problematic. Studies are therefore urgently needed to 1) establish more complete epidemiological surveillance of CVD trends in LIC; 2) determine a CVD risk calculator that is specifically calibrated to populations from these countries; 3 ) assess the benefits, harms, compliance, and adherence to long-term aspirin therapy in these populations; and 4) undertake a cost-to-benefit comparison between aspirin therapy and alternatives such as clopidogrel or statins in these settings. Before such evidence is available in LIC, aspirin should not be routinely prescribed in primary cardiovascular prevention, but could be considered in patients with very high cardiovascular risk and without risk factors for gastrointestinal and intracranial bleeding.

\section{Author contributions}

Both authors contributed to conception of the paper, drafting and critically revising the paper, and agree to be accountable for all aspects of the work.

\section{Disclosure}

The authors report no conflicts of interest in this work.

\section{References}

1. Lim SS, Vos T, Flaxman AD, et al. A comparative risk assessment of burden of disease and injury attributable to 67 risk factors and risk factor clusters in 21 regions, 1990-2010: a systematic analysis for the Global Burden of Disease Study 2010. Lancet. 2012;380: 2224-2260

2. World Health Organization. Atlas of heart disease and stroke. [cited February 01, 2015]. Available from: http://www.who.int/cardiovascular_diseases/resources/atlas/en/. Accessed August 7, 2015.

3. World Health Organization. Neglected global epidemics: three growing threats. In: Shaping the Future. The World Health Report. Geneva: World Health Organization; 2003:85-95. 
4. Mendis S, Al Bashir I, Dissanayake L, et al. Gaps in capacity in primary care in low-resource settings for implementation of essential noncommunicable disease interventions. Int J Hypertens. 2012;2012:584041.

5. Biondi-Zoccai G, Wu Y, Serrano CV Jr, Frati G, Agostoni P, Abbate A. Aspirin underuse, non-compliance or cessation: definition, extent, impact and potential solutions in the primary and secondary prevention of cardiovascular disease. Int J Cardiol. 2014;182C:148-154.

6. Cohen AT, Imfeld S, Markham J, Granziera S. The use of aspirin for primary and secondary prevention in venous thromboembolism and other cardiovascular disorders. Thromb Res. 2015;135(2):217-225.

7. Antithrombotic Trialists' (ATT) Collaboration; Baigent C, Blackwell L, et al. Aspirin in the primary and secondary prevention of vascular disease: collaborative meta-analysis of individual participant data from randomised trials. Lancet. 2009;373:1849-1860.

8. Vandvik PO, Lincoff AM, Gore JM, et al. Primary and secondary prevention of cardiovascular disease: antithrombotic therapy and prevention of thrombosis, 9th: American College of Chest Physicians Evidence-Based Clinical Practice Guidelines. Chest. 2012;141(2 Suppl):637S-668S.

9. Bartolucci AA, Tendera M, Howard G. Meta-analysis of multiple primary prevention trials of cardiovascular events using aspirin. Am J Cardiol. 2011;107:1796-1801.

10. Raju N, Sobieraj-Teague M, Hirsh J, O’Donnell M, Eikelboom J. Effect of aspirin on mortality in the primary prevention of cardiovascular disease. Am J Med. 2011;124:621-629.

11. Pignone M, Alberts MJ, Colwell JA, et al. Aspirin for primary prevention of cardiovascular events in people with diabetes: a position statement of the American Diabetes Association, a scientific statement of the American Heart Association, and an expert consensus document of the American College of Cardiology Foundation. Circulation. 2010;121:2694-2701.

12. De Berardis G, Lucisano G, D'Ettorre A, et al. Association of aspirin use with major bleeding in patients with and without diabetes. JAMA. 2012;307:2286-2294.

13. Ikeda Y, Shimada K, Teramoto T, et al. Low-dose aspirin for primary prevention of cardiovascular events in Japanese patients 60 years or older with atherosclerotic risk factors: a randomized clinical trial. JAMA. 2014;312:2510-2520

14. Sutcliffe P, Connock M, Gurung T, et al. Aspirin for prophylactic use in the primary prevention of cardiovascular disease and cancer: a systematic review and overview of reviews. Health Technol Assess. 2013;17(43):1-253.
15. Depta JP, Bhatt DL. Should patients stop taking aspirin for primary prevention? Cleve Clin J Med. 2015;82(2):91-96.

16. Battistoni A, Mastromarino V, Volpe M. Reducing cardiovascular and cancer risk: how to address global primary prevention in clinical practice. Clin Cardiol. 2015;38(6):387-394.

17. Halvorsen S, Andreotti F, ten Berg JM, et al. Aspirin therapy in primary cardiovascular disease prevention: a position paper of the European Society of Cardiology working group on thrombosis. J Am Coll Cardiol. 2014;64(3):319-327.

18. GBD 2013 Mortality and Causes of Deaths Collaborators. Global, regional, and national age-sex specific all-cause and cause-specific mortality for 240 causes of death, 1990-2013: a systematic analysis for the Global Burden of Disease Study 2013. Lancet. 2015;385(9963): 117-171.

19. Ridker PM, Cook NR, Lee IM, et al. A randomized trial of low-dose aspirin in the primary prevention of cardiovascular disease in women. N Engl J Med. 2005;352(13):1293-1304.

20. DeFilippis AP, Young R, Carrubba CJ, et al. An analysis of calibration and discrimination among multiple cardiovascular risk scores in a modern multiethnic cohort. Ann Intern Med. 2015;162(4):266-275.

21. Yusuf S, Hawken S, Ounpuu S, et al. Effect of potentially modifiable risk factors associated with myocardial infarction in 52 countries (the INTERHEART study): case-control study. Lancet. 2004;364: 937-952.

22. McGorrian C, Yusuf S, Islam S, et al. Estimating modifiable coronary heart disease risk in multiple regions of the world: the INTERHEART Modifiable Risk Score. Eur Heart J. 2011;32:581-589.

23. Yusuf S, Rangarajan S, Teo K, et al. Cardiovascular risk and events in 17 low-, middle-, and high-income countries. N Engl J Med. 2014;371: 818-827.

24. Noubiap JJ, Essouma E, Bigna JJ. Targeting household air pollution for curbing the cardiovascular disease burden: a health priority in subSaharan Africa. J Clin Hypertens. Epub 2015 Jul 13.

25. Jingi AM, Noubiap JJ, Ewane Onana A, et al. Access to diagnostic tests and essential medicines for cardiovascular diseases and diabetes care: cost, availability and affordability in the West Region of Cameroon. PLoS One. 2014;9:e111812.
Vascular Health and Risk Management

\section{Publish your work in this journal}

Vascular Health and Risk Management is an international, peerreviewed journal of therapeutics and risk management, focusing on concise rapid reporting of clinical studies on the processes involved in the maintenance of vascular health; the monitoring, prevention and treatment of vascular disease and its sequelae; and the involvement of

\section{Dovepress}

metabolic disorders, particularly diabetes. This journal is indexed on PubMed Central and MedLine. The manuscript management system is completely online and includes a very quick and fair peer-review system, which is all easy to use. Visit http://www.dovepress.com/ testimonials.php to read real quotes from published authors. 